Supporting Information

\title{
Penetration of zinc into human skin
}

\section{after topical application of nano zinc}

\section{oxide used in commercial sunscreen}

\section{formulations}

\author{
Amy M. Holmesa, Ivan Kempson ${ }^{\mathrm{b},{ }^{*},}$ Tyron Tumbull ${ }^{\mathrm{b}}$, David Paterson ${ }^{\mathrm{d}}$ and Michael S. Roberts ${ }^{\mathrm{a}, \mathrm{c}^{*}}$ \\ a The University of South Australia, School of Pharmacy and Medical Sciences, Adelaide, Australia. \\ b The University of South Australia, Future Industries Institute, Mawson Lakes, Australia. \\ c The University of Queensland, Therapeutics Research Centre, Brisbane, Australia. \\ d Australian Synchrotron, Clayton, Australia. \\ * Corresponding Authors: Ivan Kempson Ivan.Kempson@unisa.edu.au; Michael Roberts \\ Michael.Roberts@unisa.edu.au
}

Table S1. Quantification of total zinc in the skin strata after dosing ZnO NPs to intact and impaired ex vivo human skin. Zinc concentrations were extracted from XRF image regions representing the Stratum corneum (SC) and viable epidermis (VE) after dosing ZnO NPs in artificial human sweat or CCT for 24 and $48 \mathrm{~h}$. Error bars indicate mean \pm 1 standard deviation or measurements run in triplicate. Data corresponds to data plotted in Figure 1 and 2. 


\begin{tabular}{|c|c|c|c|c|c|c|c|c|c|}
\hline \multirow{3}{*}{\multicolumn{2}{|c|}{$\begin{array}{l}\text { Zinc concentrations } \\
\qquad\left(\mu \mathrm{g} / \mathrm{cm}^{2}\right)\end{array}$}} & \multicolumn{4}{|c|}{ ZnO NPs applied in CCT (10\% wt) } & \multicolumn{4}{|c|}{ ZnO NPs applied in sweat ( $10 \%$ wt) } \\
\hline & & \multicolumn{2}{|c|}{$24 \mathrm{~h}$} & \multicolumn{2}{|c|}{$48 \mathrm{~h}$} & \multicolumn{2}{|c|}{$24 \mathrm{~h}$} & \multicolumn{2}{|c|}{$48 h$} \\
\hline & & Mean & SD & Mean & SD & Mean & SD & Mean & SD \\
\hline \multirow{2}{*}{ Intact } & SC & 7.288 & 1.688 & 18.670 & 8.303 & 7.619 & 2.965 & 0.458 & 0.009 \\
\hline & VE & 1.946 & 0.315 & 1.814 & 0.654 & 1.183 & 0.064 & 0.268 & 0.064 \\
\hline \multirow{2}{*}{ Impaired } & SC & 101.100 & 51.210 & 76.860 & 26.950 & 367.200 & 135.200 & 158.700 & 13.890 \\
\hline & VE & 22.510 & 2.464 & 38.530 & 5.256 & 15.420 & 1.383 & 38.940 & 5.143 \\
\hline
\end{tabular}

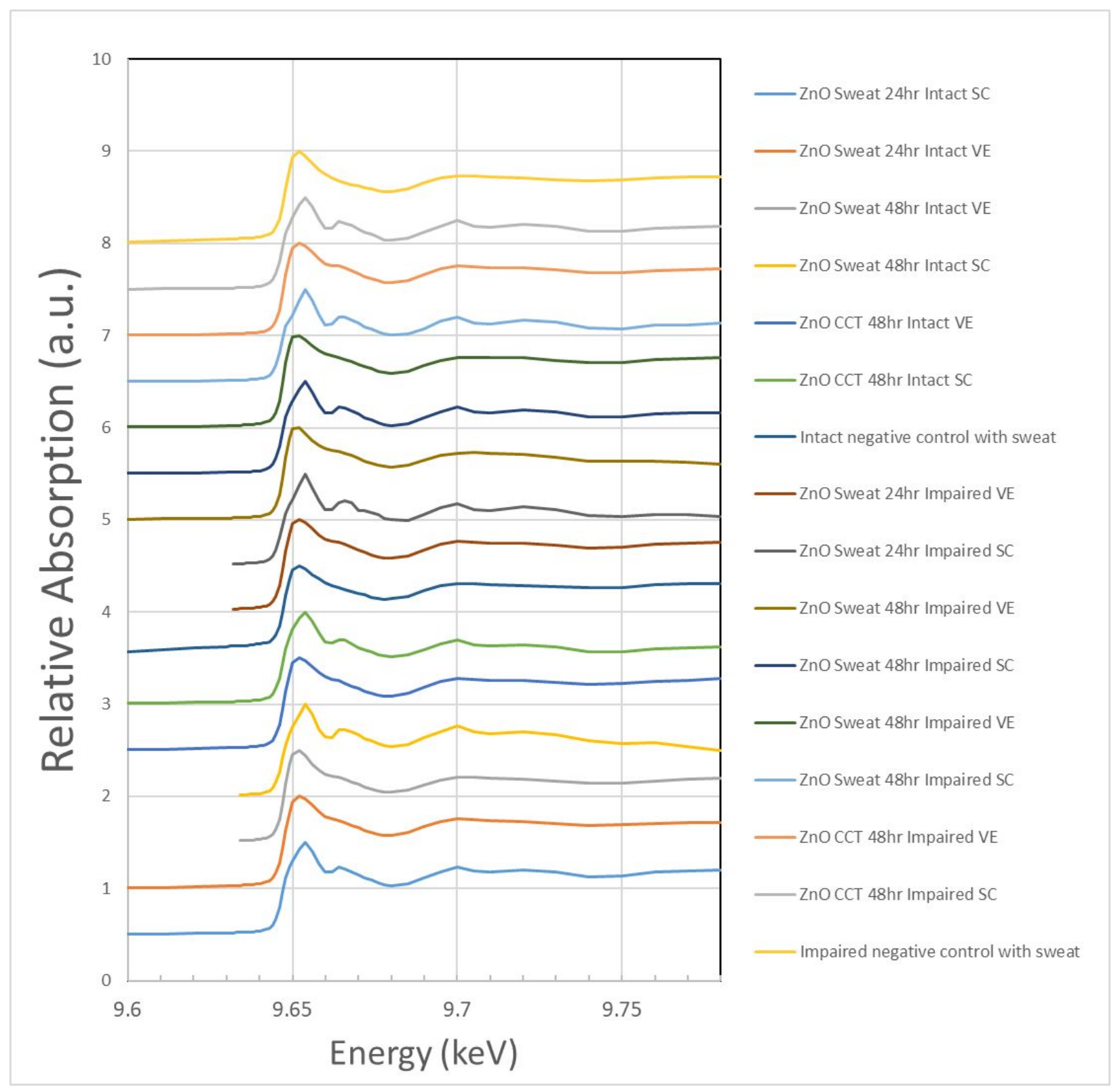

Figure S1. Normalized XANES data for each sample and region provided in Figure 4. 